\title{
The Application of the AZtec EBSD System to the Study of Strain in the SEM
}

\section{J. Goulden and A. Bewick}

Oxford Instruments NanoAnalysis, Halifax Road, High Wycombe, HP12 3SE, UK

Microstructural strain is increasingly important in materials characterisation, as any strain in a microstructure has a significant influence on both the materials properties and behavior. This presentation will consider the application of conventional-resolution EBSD to measuring strain on both the micro- and nano-scale. In addition, it will discuss detector characteristics which are important when extending conventional EBSD to high-angular resolution EBSD (HR-EBSD) using cross correlation techniques.

Strain in a grain results in small, local orientation changes; being able to detect these changes accurately is fundamental to the measurement of strain. The degree of orientation change involved is very small, so measurement requires high accuracy band detection. The main challenge for this application is in reducing "orientation noise", the error inherent in measuring crystallographic orientation, which can mask the subtle characteristics of strain. A high-precision band detection method ("AZtec Refined Accuracy") has been developed [1] which reduces orientation noise, delivering real-time $0.05^{\circ}$ orientation precision (something which is not possible using conventional, 2D Hough-based band detection, even if high Hough transform settings are used). As a result, very small shifts between patterns can be detected and subtle strain features visualized (Figure 1), offering a technique which is complementary to full HR-EBSD.

The small orientation changes detected using Refined Accuracy can be visualized and quantified using a number of different tools, both at the grain and intra-grain level. For example: Grain Orientation Spread (Figure 2) classifies grains according to their internal strain and highlights differences between grains; and there are other tools for the visualization of strain levels within a single grain, Figure 3.

A popular representation of strain levels with EBSD data is Kernal Average Misorientaion (KAM). This technique highlights very subtle deformation structures, such as low-angle boundaries, sub-grain structures and dislocation arrays. When combined with the Refined Accuracy routine this is a particularly powerful tool for highlighting subtle changes, Figure 1.

Strain analysis by EBSD benefits from the use of high quality patterns (this is particularly the case for HR-EBSD using cross correlation techniques). The EBSD detector should use a high-resolution image sensor, coupled to a high-resolution, low-distortion optic to record the patterns accurately; such a detector is the NordlysNano which has a (1344x1024) pixel sensor and virtually distortion-free optics. A relatively high sample electron dose (beam current $*$ dwell time) is required to achieve the necessary signal-to-noise ratio in the pattern. The sample dose is minimised by the use of a high-sensitivity detector. Achieving high sensitivity, high resolution and low distortion in a single detector requires the use of specialised optics, custom designed for the EBSD application, such as those in NordlysNano. 


\section{References:}

[1] K. Thomsen, N.H. Schmidt, A. Bewick, K. Larsen and J. Goulden (2013). Improving the Accuracy of Orientation Measurements using EBSD. Microscopy and Microanalysis, 19 (Suppl. 2), pp 724-725.

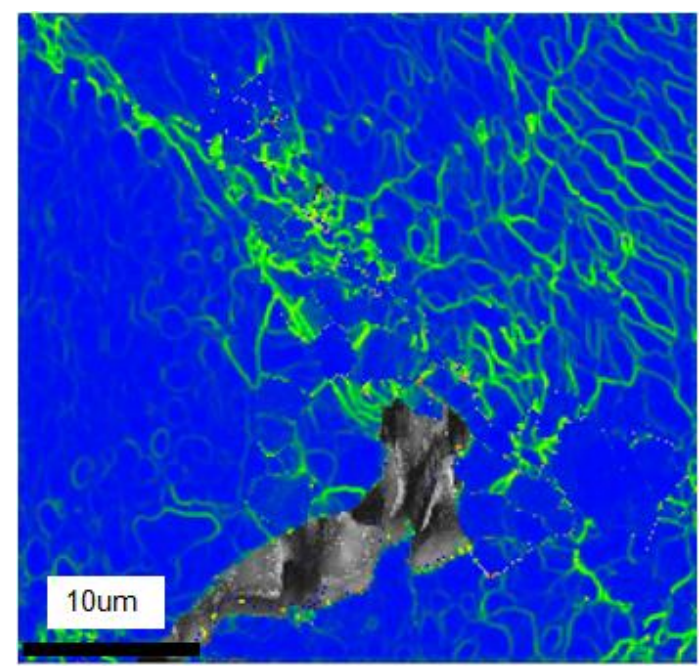

Figure 1 A crack tip in a $\mathrm{Ni}$ superalloy. The map shows a higher level of misorientation, and therefore strain, in the grains around the crack tip. This is visible as a complex pattern of deformation and the formation of sub cells of $2 \mu \mathrm{m}$ in size

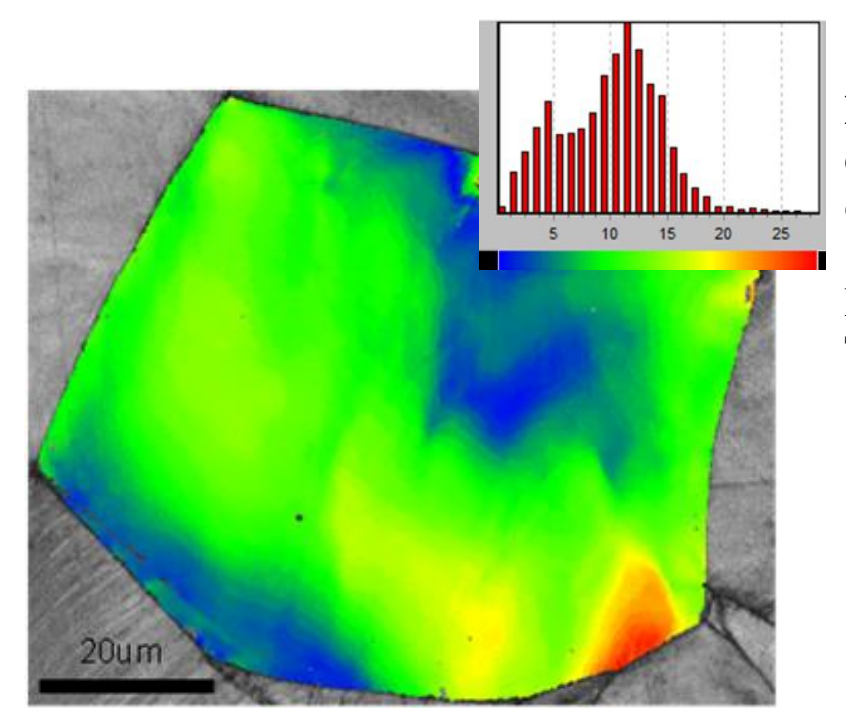

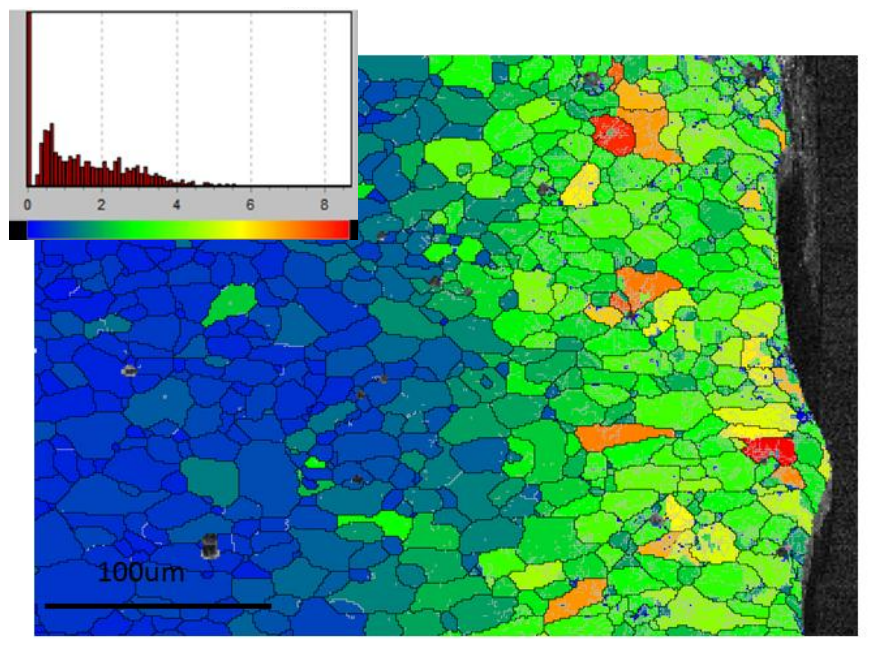

Figure 2 A shot peened aluminum in cross section. The grain orientation spread map shows those grains with a higher degree of internal strain coloured at the yellow-red end of the colour scale shown in the histrogram. These grains are closer to the shot peened surface

Figure 3 A grain in a TWIP steel showing complex internal deformation, with up to $28^{\circ}$ orientation change relative to the algorithmselected reference. Sample courtesy: Institute of Materials Science, Tampere University of Technology. 